\title{
Verifiability in Systems and Data Engineering: Preface by the Special Issue Editors
}

\author{
Agostino Cortesi ${ }^{1} \cdot$ Nabendu Chaki $^{2} \cdot$ Rituparna Chaki $^{2}$
}

Published online: 31 May 2021

(c) The Author(s), under exclusive licence to Springer-Verlag London Ltd., part of Springer Nature 2021

The pervasiveness of software systems and the growing need to guarantee their reliability makes the design and testing of methods and tools to support their verification all the more important. Besides, the verifiability of software systems needs to be trained, on the one hand, with the rapid development of data engineering techniques and, on the other hand, with the increasing complexity of cyber-physical systems that pose new security challenges.

This special issue of the journal "Innovations in Systems and Software Engineering" collects the best articles presented at the 8th International Doctoral Symposium on Applied Computation and Security Systems (ACSS 2021). Initially, each of these articles has undergone a rigorous double-blind review, evaluated by at least three referees. Subsequently, we invited the authors for the best few papers to produce extended versions of their works for this Special Issue. This was further subjected to two more rounds of reviewing by the experts in the field.

Let us briefly introduce the ten papers collected in this issue.

- "Requirement-oriented Risk Management for Incremental Software Development" by Mandira Roy et al. provides an innovative approach to the requirement management within the DevOps framework. This focuses on the treatment of conflicts among non-functional requirements to minimize the risks associated with an incorrect

$凶$ Nabendu Chaki

nabendu@ieee.org

Agostino Cortesi

cortesi@unive.it

Rituparna Chaki

rchaki@ieee.org

1 Department of Environmental Science, Informatics, and Statistics, Ca' Foscari University, Via Torino, 30172 Mestre, Venezia, Italy

2 Department of Computer Science and Engineering, University of Calcutta, Kolkata 700106, India prioritization of requirements to be incrementally developed.

- In the literature, the construction and dynamic maintenance of materialized views are carried out in a binary data space where all attributes are given the same weight. The paper "Construction and Distribution of Materialized Views in Non-Binary Data Space" by Santanu Roy et al. shows that considering different weights may be particularly significant when similar queries are fired from multiple sites in a distributed environment. Indeed, taking into account the number of actual accesses to the different attribute values may positively reflect into the ability of tuning the materialized views.

- Sabyasachee Banerjee et al. have authored the paper titled "Designing Balanced Wrapper Chains in 3D SoC under constrained TSVs". The challenge of testing the large complex 3D SoCs (3D System on Chips) in lesser time has been dealt in this work. The authors have proposed a simulated annealing-based wrapper chain design algorithm that will balance the length of the wrapper chain. The balanced length of the wrapper chains in turn reduces the total test time. Rigorous experiments on several benchmark establish that the proposed approach recorded better test lengths in more than $90 \%$ cases with an average reduction of $6.42 \%$ in test length. The proposed algorithm also used a smaller number of TSVs in most of the cases in comparable CPU time.

- The paper "Framework for end-to-end verification for Digital Microfluidics" has been written by Pushpita Roy et al. They have proposed a framework for easy specification and efficient verification of a biochemical protocol description. It operates in two stages of microfluidic protocol design, before and after synthesis. The tool developed by the authors analyzes the synthesis output for possible design errors due to incorrect synthesis. Experimental results establish the utility of the proposed framework for verifiable protocol design in digital microfluidics. 
- A metaheuristic discrete genetic-learning-enabled particle swarm optimization algorithm has been proposed by Gouri Kundu et al. in the paper titled "A Discrete Genetic Learning enabled PSO for Targeted Positive Influence Maximization in Consumer Review Networks". This is combined with a trustworthiness-heuristic-based local search strategy for targeted positive influence maximization in consumer review networks (CRN). The existing spread estimation function has been replaced by a computationally efficient positive influence spread estimation function. The improvement in solution accuracy (measured in terms of positive influence spread) achieved by the proposed D-GLPSO-TPIM algorithm has been verified by comparing it with the existing state of the art.

- Arnab Kumar Maji et al. have written "A Novel Hybrid Genetic Algorithm based Firefly Mating Algorithm for Solving Sudoku". The authors have presented a hybrid genetic algorithm-based firefly mating algorithm. This can solve Sudoku instances with a greater success rate for easy, medium, and hard difficulty level puzzles. Even though the success rate of this algorithm is much greater in comparison with other existing works, this may result in optimized execution time and effective memory space utilization. The proposed method has reached beyond local optima for a smaller population and lesser generation.

- Kaushik Roy et al. have proposed a new CNN-based approach for script detection- "STDNet:A CNN based approach to single/mixed script detection". In this work, a CNN-based deep learning framework, called STDNet (Script-Type detection Network), has been proposed to detect single/mixed script images. Single script and also mixed script classes were regarded in the collection of images with-outlier and without-outlier sets constructed for this work. In order to test the robustness as well as to have an increased volume of the dataset, noises were added to the original dataset. The authors have claimed to attain an overall maximum accuracy of $99.53 \%$ without any outlier in the data set. The accuracy of $99.93 \%$ was obtained for the single-script class in a normal scenario. The accuracy of the proposed network was compared with two of the existing approaches.

- Srijoni Majumdar et al. have proposed a new mathematical framework extending her previous experimental study presented during the ACSS 2021 symposium. The paper is titled as "A Mathematical Framework for Design Discovery from Multi-threaded Applications using Neural Sequence Solvers". The authors have proposed a generic mathematical model to interpret run-time non-deterministic events and encode functional as well as thread-specific behavior in the form of quantifiable features. This can be fitted into a standard solver for automated inference of design aspects from multi-threaded applications. A tool named Dcube has been built based on the mathematical model. It uses various classifiers of a machine learning framework for concurrency and resource management-related issues.

- Maciej Szymkowski et al. have authored the paper "Iris-based Human Identity Recognition with Machine Learning methods and Discrete Fast Fourier Transform". The authors aims toward proposing a low-computation cost and accurate iris-based human recognition system that can be implemented without using any specialized devices. Discrete fast Fourier transform is selected by principal component analysis (PCA) algorithm. Three algorithms have been used for classification. The most precise result, with 98\% accuracy, has been observed for support vector machines (SVM) algorithm. However, the authors have also explored KNN and artificial neural network and established their usefulness in the actual deployment. Each classification algorithm was tested with the database consisting of 510 samples.

- The final chapter is a contribution by Kamal Das et al. titled "Parallel Simulation of Cyber-Physical Systems". The authors proposed a parallel simulation engine for hybrid automaton models of Cyber-Physical-Systems (CPS), which can compute random simulations in parallel on multicore processors. This is based on a mathematical model of the system to virtually execute and test CPS systems via model simulations to understand the system dynamics better. The authors demonstrated the performance gains of the proposed simulation engine over SpaceEx and CORA, the modern model checkers and simulators for affine hybrid systems. The simulation implements a multithreaded lock-free algorithm to compute and compare.

We are deeply grateful to Mike Hinchey for his strong support toward the delivery of this special issue, and to all the colleagues who has served as reviewers giving the authors valuable suggestions to improve the overall quality of their work, and thereby enriching this Special Issue.

Publisher's Note Springer Nature remains neutral with regard to jurisdictional claims in published maps and institutional affiliations. 